\title{
ESTUDO COMPARATIVO DAS TÉCNICAS RADIOGRÁFICAS E DOSES ENTRE $O$ BRASIL E A AUSTRÁLIA*
}

\author{
Ana Cecília Pedrosa de Azevedo ${ }^{1}$, Kouther Elhaj Mohamed Mohamadain ${ }^{2}$, Otolorin \\ Adelaja Osibote ${ }^{3}$, Arnaldo Levy Lassance Cunha ${ }^{4}$, Antonio Pires Filho ${ }^{5}$
}

Resumo OBJETIVO: O objetivo deste trabalho é fazer um estudo comparativo das técnicas radiográficas entre o Brasil e a Austrália e avaliar as doses de radiação para os exames radiológicos mais comuns. MATERIAIS E MÉTODOS: A dose de entrada na pele (DEP) e a dose efetiva (DE) foram obtidas com o uso do programa DoseCal. Em ambos os países, o estudo foi realizado em hospitais de grande porte da rede pública. Quatro tipos de exames foram avaliados: tórax, abdome, pelve e coluna torácica em três projeções (ântero-posterior, póstero-anterior e lateral). Na Austrália, todos os equipamentos são digitais e operam no sistema PACS. No Brasil, os aparelhos são convencionais. RESULTADOS: Os valores médios da DEP e da DE são consideravelmente mais altos no Brasil, exceto para os exames de tórax, cujos resultados indicaram índices menores. As maiores diferenças encontradas foram para os exames de pelve ( 26 vezes maior no Brasil) e de coluna torácica (43 vezes maior no Brasil). 0 mesmo acontece para os valores da DE. CONCLUSÃO: Nos hospitais australianos, os programas de controle e garantia de qualidade (PCGQ) fazem parte da rotina nos serviços de radiologia. Contam com equipamentos digitais de última geração e os serviços possuem uma equipe de física médica atuante. Esse conjunto de iniciativas resulta na produção de imagens radiográficas de alta qualidade, com baixas doses e índices de rejeição próximos a zero. Tais resultados apontam para a necessidade de se estimular a implantação de PCGO em toda a rede hospitalar brasileira. No entanto, analisando os resultados de DEP nos exames de tórax, concluímos que doses baixas também são possíveis no Brasil se forem empregadas técnicas radiográficas adequadas.

Unitermos: Dose de entrada na pele; Dose efetiva; Radiodiagnóstico; Dosimetria.

Abstract Comparison between radiological techniques and doses used in Brazil and in Australia.

OBJECTIVE: To compare radiological techniques used in Brazil and in Australia, and to evaluate radiation doses of the most common $x$-ray procedures. MATERIALS AND METHODS: The entrance skin dose (ESD) and the effective dose (ED) were obtained with the software DoseCal. In both countries the study was conducted in large public hospitals. Four distinct procedures were evaluated: chest, abdomen, pelvis and thoracic spine in three views (anterior-posterior, posterior-anterior and lateral) In Australia, all x-ray equipment are digital and linked with PACS whereas in Brazil conventional equipment is used. RESULTS: The mean values of ESD and ED were consistently higher in Brazil, except for chest x-rays. The higher differences were seen in pelvis ( 26 fold higher in Brazil) and thoracic spine (43 fold higher in Brazil) radiographs. CONCLUSION: All Australian hospitals have quality assurance programs (QAP) implemented on a permanent basis, the most advanced digital equipment and count with an effective medical physics service. These factors are determinant for high quality images, low rejection rates and low doses imparted to patients when compared to Brazilian standards. Theses results reinforce the need of implementation of quality assurance programs in Brazil. However, when analyzing the results of ESD for chest $x$-rays, we concluded that low doses can also be achieved in Brazil with the use of appropriate $x$-ray techniques.

Key words: Entrance skin dose; Radiodiagnosis; Effective dose; Dosimetry.

* Trabalho realizado na Fundação Oswaldo Cruz (Fiocruz), Rio de Janeiro, RJ.

1. Física, D.Sc., Fiocruz - Escola Nacional de Saúde Pública Sérgio Arouca/CESTEH.

2. Física, M.Sc., Sudan University of Science and Technology, Faculty of Sciences, Physics Department, Khartoum, Sudan (em doutorado-"sanduíche" no Brasil).

3. Física, M.Sc., Ogun State University, Nigeria, e Third World Organization for Women in Science/ICTP, Trieste, Itália (em doutorado-"sanduíche" no Brasil).

4. Médico Sanitarista, Fiocruz - Escola Nacional de Saúde Pública Sérgio Arouca/CESTEH, Secretaria de Estado de Saúde do Rio de Janeiro, Centro de Vigilância Sanitária e Laboratório de Ciências Radiológicas, Universidade do Estado do Rio de Janeiro (UERJ).

5. Serviço de Radiologia, Hospital Geral de Bonsucesso.

Endereço para correspondência: Dra. Ana Cecília Pedrosa de

\section{INTRODUÇÃO}

No Brasil, em 1998, foi publicada a Portaria no $453 / 98^{(\mathbf{1})}$, que estabelece as "Diretrizes de proteção radiológica em radiodiagnóstico médico e odontológico". Desde então, todo estabelecimento médi-

Azevedo. Fundação Oswaldo Cruz - Escola Nacional de Saúde Pública Sérgio Arouca/CESTEH. Rua Leopoldo Bulhões, 1480, Manguinhos. Rio de Janeiro, RJ, 21041-210. E-mail: acpa@ ensp.fiocruz.br

Recebido para publicação em 24/6/2004. Aceito, após revisão, em 7/1/2005. co que faça uso de raios $\mathrm{X}$ como método diagnóstico deverá seguir o estabelecido nessa Portaria. A principal contribuição dessa norma técnica é a exigência da implantação de programas de controle e garantia de qualidade (PCGQ) em serviços de radiodiagnóstico.

A Organização Mundial da Saúde (OMS) define garantia de qualidade em radiologia diagnóstica como sendo "um esforço organizado da direção do departamento no sentido de garantir que sejam 
produzidas imagens de qualidade suficiente para fornecer um diagnóstico adequado com a menor dose para o paciente".

Existe extensa literatura sobre a implantação de PCGQ em radiodiagnóstico e avaliação de doses em pacientes ${ }^{(\mathbf{2 - 1 0})}$. Os PCGQ incluem testes periódicos nos equipamentos e acessórios e a revisão sistemática dos procedimentos (administrativos, de manutenção preventiva e de treinamento), tendo como principal objetivo garantir uma imagem de alta qualidade com a menor dose possível para o paciente e um baixo custo associado.

\section{MATERIAIS E MÉTODOS}

O Hospital Geral de Bonsucesso (HGB) é um hospital público brasileiro, federal, de grande porte. $\mathrm{O}$ serviço de radiodiagnóstico possui apenas equipamentos radiológicos convencionais.

$\mathrm{Na}$ Austrália, os dados também foram obtidos em três hospitais de grande porte da rede pública federal: o Westmead Hospital, em Sydney, e o The Royal Brisbane Hospital e o The Prince Charles Hospital, em Brisbane. Todos os equipamentos desses hospitais são digitais, de última geração, e trabalham com o sistema de armazenamento de imagens PACS ("picture archiving and communication systems"). Este recurso permite a integração e troca de imagens entre todos os hospitais da rede pública australiana.

Em todos os hospitais os dados antropométricos dos pacientes (idade, sexo, peso) e a técnica radiográfica $(\mathrm{kVp}, \mathrm{mAs}$, DFP) foram registrados. Na avaliação das doses, apenas os exames que tiveram imagens aceitas para diagnóstico foram incluídos na pesquisa. Este procedimento garante que os valores encontrados sejam representativos das doses. Quatro tipos de exames foram avaliados: tórax, abdome, pelve e coluna torácica em três projeções (ântero-posterior (AP), póstero-anterior (PA), e lateral (LAT)).

O método mais usual para se avaliar a dose de entrada na pele (DEP) envolve o uso de dosímetros termoluminescentes (TLD). No entanto, o uso desses dosímetros é trabalhoso e consome muito tempo, sendo, portanto, desaconselhado para estudos que envolvam grande número de pacientes. Os TLD têm sido gradualmente substituídos por métodos mais modernos e mais baratos e que produzem resultados mais confiáveis.

Nesse sentido, o uso de programas computacionais para a avaliação da DEP e da dose efetiva (DE) é um recurso moderno de dosimetria ${ }^{(\mathbf{1 1 , 1 2})}$ que está sendo largamente utilizado em hospitais no mundo inteiro. O programa usado nesta pesquisa foi especialmente desenvolvido para avaliar a DEP e a DE que os pacientes recebem quando são submetidos aos exames radiológicos mais comuns. O programa chama-se DoseCal e foi desenvolvido no Radiological Protection Centre do Saint George's Hospital em Londres e generosamente cedido para este trabalho de pesquisa. Em trabalho anterior ${ }^{(\mathbf{1 3})}$ testamos a validade/eficiência desse programa, que demonstrou grau de confiabilidade maior que $90 \%$.

O desenvolvimento de métodos práticos para a avaliação de doses em pacientes é de extrema importância e faz parte dos PCGQ que devem ser implantados em toda instituição médica que faça uso de radiações ionizantes. No Brasil, a Portaria no 453/98 ${ }^{(1)}$ é o instrumento legal para esse fim.

Para que o DoseCal forneça os valores de DEP e DE é necessário que sejam inseridos no programa alguns valores, dentre eles o rendimento do tubo de raios $\mathrm{X}$ utilizado na tomada radiográfica. Esse valor é obtido usando-se uma câmara de ionização devidamente calibrada. Os valores de DEP são fornecidos pela equação abaixo $^{(14)}$, onde $\mathrm{kV}$ é a quilovoltagem do tubo, mA é a miliamperagem, s é o tempo de exposição em milissegundos, FRE é o fator de retroespalhamento e DFP é a distância foco-pele. Nesta equação o rendimento é o rendimento do tubo obtido com $80 \mathrm{kV}$ e a uma distância de $1 \mathrm{~m}$ normalizado para $10 \mathrm{mAs}$.

$$
\mathrm{DEP}=\text { rendimento } \mathrm{x}\left(\frac{\mathrm{kV}}{80}\right)^{2} \times\left(\frac{100}{\mathrm{DFP}}\right)^{2} \times \frac{\mathrm{mAs}}{\mathrm{FRE}}
$$

A página inicial do programa DoseCal está exemplificada na Figura 1. Nela são inseridos os valores do rendimento do tubo, a identificação do paciente e os valores da técnica radiográfica empregada no exame (kVp, mAs e DFP). O tipo de exa- me, assim como a projeção e a filtração, são selecionados a seguir.

Os tipos de exame disponíveis no programa são os listados nas publicações da National Radiological Protection Board (NRPB) para adultos e crianças ${ }^{(15,16)}$. As tabelas da NRPB contêm os coeficientes de conversão que são utilizados pelo programa no cálculo da DEP e da DE.

Quando a página inicial estiver completa, o usuário deve pressionar o botão CALC DOSES. Os resultados aparecem imediatamente, como se vê na Figura 2.

Neste trabalho, os pacientes não precisaram ser identificados, pois o objetivo da pesquisa foi obter valores médios representativos de doses para os diversos tipos de exames praticados nos hospitais. Mesmo assim, o projeto foi submetido e aprovado pelo Comitê de Ética em Pesquisa da Escola Nacional de Saúde Pública Sérgio Arouca da Fundação Oswaldo Cruz, tendo termo de autorização assinado pelo chefe do Serviço de Radiologia do HGB.

\section{RESULTADOS}

Na Tabela 1 é apresentado o tratamento estatístico dos dados de DEP (mGy) e DE (mSv) para tórax, abdome, pelve e coluna torácica. São apresentados os valores máximos e mínimos, o desvio-padrão (DP) e a mediana. $\mathrm{O}$ hospital brasileiro apresenta 155 pacientes e os australianos, 408.

A Tabela 2 apresenta os valores dos parâmetros de exposição utilizados na Austrália e no Brasil. Os valores médios encontram-se entre parênteses.

Pode-se observar que, na Austrália, é utilizada a técnica de alta quilovoltagem ${ }^{\mathbf{( 1 6}}$ para todos os exames. Para tórax PA, por exemplo, o valor médio é de $101 \mathrm{kV}$, enquanto no Brasil esse valor é de apenas 73 $\mathrm{kV}$. Conseqüentemente, os valores médios de mAs têm que ser muito mais altos no Brasil (10 mAs) do que na Austrália (4-5 mAs). A DFP na Austrália é de $180 \mathrm{~cm} \mathrm{e}$ no Brasil é de $150 \mathrm{~cm}$. Mesmo assim, os valores brasileiros nos exames de tórax estão um pouco abaixo dos valores australianos, o que demonstra que há outros fatores influenciando no valor final das doses. No Brasil, para os outros exames (abdome, pelve e coluna torácica), a técnica radiográfica empregada também é de baixa 


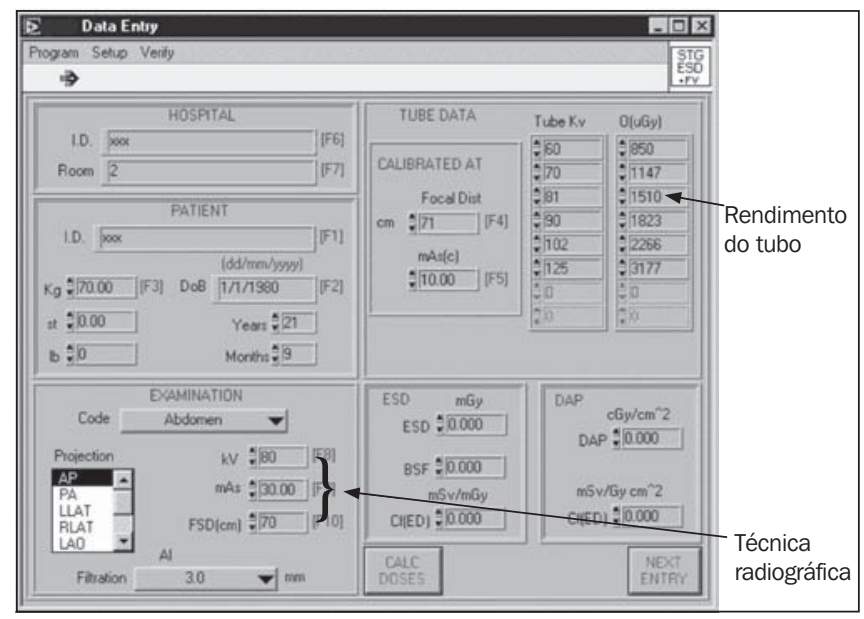

Figura 1. Página inicial do DoseCal.

Tabela 1 Tratamento estatístico e valores de DEP e DE nos quatro hospitais.

\begin{tabular}{|c|c|c|c|c|c|c|}
\hline Hospital & \multicolumn{2}{|c|}{ Westmead } & \multicolumn{2}{|c|}{ Royal Brisbane } & \multicolumn{2}{|c|}{ Prince Charles } \\
\hline Exames/Projeção & Tórax PA & Tórax LAT & Tórax PA & Abdome AP & Pelve AP & Coluna torácica LAT \\
\hline \multicolumn{7}{|l|}{ DEP (mGy) } \\
\hline Média & 0,14 & 0,48 & 0,08 & 0,20 & 0,07 & 0,04 \\
\hline Mínima & 0,06 & 0,14 & 0,04 & 0,12 & 0,04 & 0,02 \\
\hline Máxima & 0,45 & 1,41 & 0,14 & 0,37 & 0,09 & 0,07 \\
\hline Desvio-padrão & 0,05 & 0,30 & 0,02 & 0,07 & 0,02 & 0,02 \\
\hline Mediana & 0,12 & 0,34 & 0,07 & 0,16 & 0,07 & 0,03 \\
\hline № de pacientes & 34 & 24 & 45 & 45 & 4 & 3 \\
\hline $\mathrm{DE}(\mathrm{mSv})$ & 0,02 & 0,04 & 0,01 & 0,02 & 0,01 & 0,01 \\
\hline Hospital & \multicolumn{6}{|c|}{ Hospital Geral de Bonsucesso } \\
\hline Exames/Projeção & Tórax PA & Tórax LAT & & Abdome AP & Pelve AP & Coluna torácica LAT \\
\hline DEP (mGy) & & & & & & \\
\hline Média & 0,10 & 0,33 & & 1,47 & 1,87 & 1,71 \\
\hline Mínimo & 0,03 & 0,02 & & 0,05 & 0,39 & 0,18 \\
\hline Máximo & 0,29 & 4,66 & & 4,87 & 4,77 & 6,30 \\
\hline Desvio-padrão & 0,03 & 0,17 & & 1,04 & 0,84 & 1,20 \\
\hline Mediana & 0,09 & 0,25 & & 0,93 & 1,40 & 1,06 \\
\hline № de pacientes & 215 & 89 & & 25 & 21 & 13 \\
\hline $\mathrm{DE}(\mathrm{mSv})$ & 0,01 & 0,03 & & 0,19 & 0,28 & 0,04 \\
\hline
\end{tabular}

DEP, dose de entrada na pele; DE, dose efetiva; PA, póstero-anterior; LAT, lateral; AP, ântero-posterior.

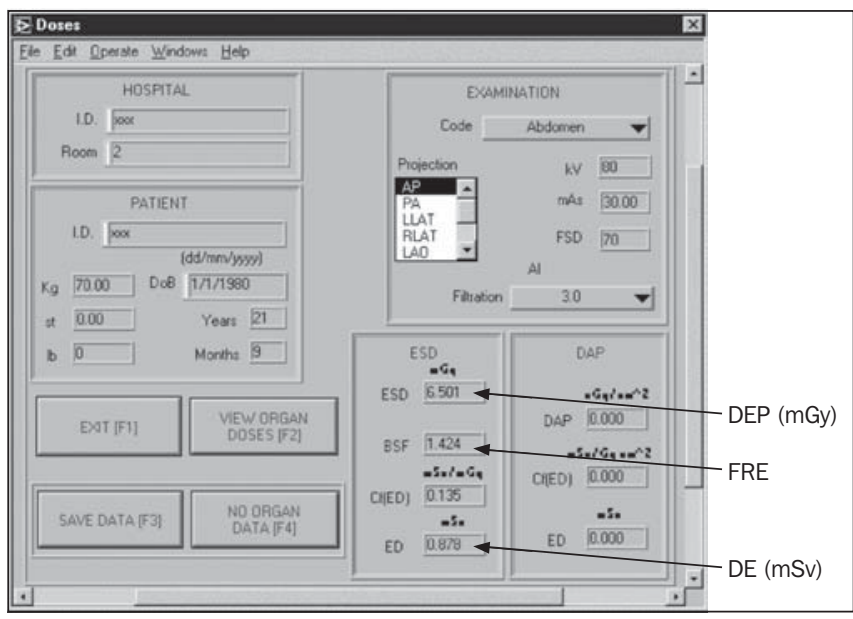

Figura 2. Página de resultados do DoseCal.

quilovoltagem, como pode ser visto na Tabela 2. Baixa $\mathrm{kV}$ associado a alta $\mathrm{mAs}$ e DFP reduzida, que resultaram em altas doses fornecidas aos pacientes.

Pode-se observar que, no Brasil, a quilovoltagem varia de acordo com a espessura do paciente. Para tórax PA, por exemplo, varia entre 48 e $100 \mathrm{kV}$, e para tórax LAT varia entre 70 e 117 kV. Na Austrália a quilovoltagem é praticamente fixa e o que varia é apenas a carga transportável (mAs). Como se pode ver na Tabela 2, nos exames de tórax, a quilovoltagem variou de 100 a 102 para projeção em PA e de 117 a 120 para projeção LAT (no Westmead Hospital). No The Royal Brisbane Hospital se manteve fixa $(102 \mathrm{kV})$.

A Tabela 2 exemplifica a falta de padronização, no Brasil, com respeito à tecnica radiográfica. No entanto, o fator mais importante para o aumento da dose parece estar associado ao valor na carga transportável empregada.

Tabela 2 Fatores de exposição utilizados nos quatro hospitais.

\begin{tabular}{|c|c|c|c|c|c|c|}
\hline \multirow{2}{*}{$\begin{array}{l}\text { Hospital } \\
\text { Exames/Projeção }\end{array}$} & \multicolumn{2}{|c|}{ Westmead } & \multicolumn{2}{|c|}{ Royal Brisbane } & \multicolumn{2}{|c|}{ Prince Charles } \\
\hline & Tórax PA & Tórax LAT & Tórax PA & Abdome AP & Pelve AP & Coluna torácica LAT \\
\hline $\begin{array}{l}\mathrm{kVp} \\
\mathrm{mAs} \\
\text { DFP (cm) }\end{array}$ & $\begin{array}{c}100-102(101) \\
2-16(5) \\
180\end{array}$ & $\begin{array}{c}117-120(117) \\
4-41(14) \\
180\end{array}$ & $\begin{array}{c}102 \\
2,8-5,0 \\
100\end{array}$ & $\begin{array}{c}70 \\
13-32(22) \\
100\end{array}$ & $\begin{aligned} 74-80 & (77) \\
18-44 & (33) \\
90-115 & (104)\end{aligned}$ & $\begin{array}{c}70-125(90) \\
5-61(28) \\
100-140(123)\end{array}$ \\
\hline Hospital & \multicolumn{6}{|c|}{ Hospital Geral de Bonsucesso } \\
\hline Exames/Projeção & Tórax PA & Tórax LAT & & Abdome AP & Pelve AP & Coluna torácica LAT \\
\hline $\begin{array}{l}\mathrm{kVp} \\
\mathrm{mAs} \\
\text { DFP }(\mathrm{cm})\end{array}$ & $\begin{array}{c}48-100(73) \\
3-18(10) \\
100-174(150)\end{array}$ & $\begin{array}{c}70-117(85) \\
7-42(19) \\
68-165(142)\end{array}$ & & $\begin{array}{cc}59-96 & (74) \\
20-100 & (47) \\
78-140 & (97)\end{array}$ & $\begin{array}{c}62-81(70) \\
18-100(52) \\
83-95(89)\end{array}$ & $\begin{array}{c}57-77 \quad(69) \\
39-100 \quad(62) \\
78-106(87)\end{array}$ \\
\hline
\end{tabular}

DFP, distância foco-pele; PA, póstero-anterior; LAT, lateral; AP, ântero-posterior. 
O índice de rejeição ${ }^{(\mathbf{1 7}, 18)}$ no hospital brasileiro é de aproximadamente $15 \%$, enquanto na Austrália é próximo de zero. Este resultado é parcialmente explicado pelo fato que, na Austrália, em se tratando de equipamentos digitais, é possível ajustar o nível de contraste posteriormente à tomada radiográfica, evitando assim a repetição das radiografias.

Os valores de DE encontram-se todos abaixo dos limites ${ }^{(2,3)}$ internacionais recomendados.

\section{DISCUSSÃO}

Os hospitais australianos apresentaram as menores doses, exceto para os exames de tórax, nos quais os resultados brasileiros são um pouco mais baixos.

Esse resultado é devido a vários fatores: exposição, técnica radiográfica, peso do paciente, DFP, filtração, idade, tipo e manutenção dos aparelhos e treinamento dos profissionais que atuam no serviço.

Aumentos de doses de até 43 vezes foram encontrados. As doses altas estão quase sempre associadas a valores elevados de mAs.

Existe grande potencial para redução das doses, não necessariamente associado a altos investimentos. Um exemplo é o fato de que mesmo com emprego de equipamentos modernos digitais, os hospitais australianos não obtiveram as menores doses para os exames de tórax. No caso do Brasil, este estudo aponta para o fato de que a simples mudança dos valores da técnica radiográfica poderia em muito contribuir para uma redução significativa das doses nos exames de abdome e pelve. Em nosso país a dose não é considerada fator limitante, sendo o principal fator a qualidade da imagem radiográfica. Na maioria das vezes, os próprios médicos desconhe- cem a dose que o seu paciente está recebendo, uma vez que sua avaliação ainda é pouco difundida em nosso país. Altos valores de mAs são empregados para a obtenção de um bom contraste. Esta filosofia deveria ser modificada no sentido de manter as doses tão baixas quanto razoavelmente exeqüíveis (princípio ALARA "As Low As Reasonably Achievable").

A implantação de PCGQ é um recurso importante para a melhoria da prestação de serviços radiológicos à população e otimização da prática radiológica.

\section{Agradecimentos}

Os autores agradecem ao Radiological Protection Centre - Saint George's Hospital (Londres), pelo uso do programa DoseCal, ao Dr. Mourão Filho, chefe do Serviço de Radiologia do Hospital Geral de Bonsucesso, por seu apoio à realização dessa pesquisa, e às seguintes instituições: Universidade Federal do Rio de Janeiro, Third World Organization for Women in Science (Trieste, Itália), International Centre for Theoretical Physics (Trieste, Itália), Fundação Oswaldo Cruz (Fiocruz), Laboratório de Ciências Radiológicas da Universidade do Estado do Rio de Janeiro e Centro de Vigilância Sanitária da Secretaria de Estado de Saúde do Rio de Janeiro.

\section{REFERÊNCIAS}

1. Brasil. Ministério da Saúde. Diretrizes de proteção radiológica em radiodiagnóstico médico e odontológico. Portaria no 453. Diário Oficial da União 103, 1/6/1998.

2. National Radiological Protection Board. Patient dose reduction in diagnostic radiology. Documents of the NRPB 1990;1(3). London, UK: HMSO.

3. National Radiological Protection Board. Guidelines on patient dose to promote the optimization of protection for diagnostic medical exposures. Documents of the NRPB 1999;10(1).

4. International Commission on Radiological Protection. Recommendations of the International Commission on Radiological Protection. ICRP Publication 60. Oxford: Pergamon Press, 1991.
5. International Commission on Radiological Protection. Recommendations of the International Commission on Radiological Protection. ICRP Publication 73. Oxford: Pergamon Press, 1996.

6. International Commission on Radiological Protection. Recommendations of the International Commission on Radiological Protection. ICRP Publication 26. Oxford: Pergamon Press, 1977.

7. Commission of the European Communities. Quality criteria for diagnostic radiographic images. Working Document CEC No. XII/173/90. 2nd ed. June 1990.

8. United Nations Scientific Committee on the Effects of Atomic Radiation. Sources effects and risks of ionizing radiation. UNSCEAR 2000 Report, vol. II: Effects. New York, NY: United Nations, 2000.

9. NRPB IPSM COR. National protocol for patient dose measurements in diagnostic radiology. Chilton, UK: NRPB, 1992.

10. Council Directive 97/43/Euratom of 30 June 1997 on Health Protection of Individuals Against the Dangers of Ionizing Radiation in Relation to Medical Exposure. Official Journal of the European Communities No. L180, 1997.

11. Kyriou JC, Newey V, Fitzgerald MC. Patient doses in diagnostic radiology at the touch of a button. London, UK: St. George's Hospital, 2000.

12. Cook V, Shah K, Pablot S, Kyriou JC. A practice in the X ray imaging of children. London, UK: St. George's Hospital \& St. Helier Hospital, 1998.

13. Mohamadain KEM, Azevedo ACP, Rosa LAR, Guebel MRN, Boechat MCB. Dose measurements using thermoluminescent dosimeters and DoseCal software at two paediatric hospitals in Rio de Janeiro. Appl Radiat Isot 2003;59:53-57.

14. Davies M, McCallum H, White G, Brown J, Hlem M. Patient dose audit in diagnostic radiography using custom designed software. RadioGraphics 1997;3:17-25.

15. Hart D, Jones DG, Wall BF. Normalised organ doses for medical X-ray examinations calculated using Monte Carlo Techniques. NRPB-SR262. Chilton, UK: NRPB, 1994.

16. Hart D, Jones DG, Wall BF. Normalised organ doses for paediatric X-ray examinations calculated using Monte Carlo Techniques. NRPB-SR279. Chilton, UK: NRPB, 1996.

17. Martin CJ, Darragh CL, McKenzie GA, Bayliss AP. Implementation of a programme for reduction of radiographic doses and results achieved through increases in tube potential. Br J Radiol 1993;66:228233.

18. Azevedo ACP, Filho AP, Silva MO, Oliveira SR, Guedes LMA. Waste Cost at Rio de Janeiro Public Hospitals. World Congress on Medical Physics and Biomedical Engineering, Chicago, USA, 2000. 